\title{
Polycystic Ovary Syndrome; A Burning Issue
}

\section{Mudasir Maqbool*}

Department of Pharmaceutical Sciences, University of Kashmir, Jammu and Kashmir, India

*Corresponding Author: Mudasir Maqbool, Department of Pharmaceutical Sciences, University of Kashmir, Jammu and Kashmir, India.

Received: May 09, 2019; Published: June 07, 2019

DOI: 10.31080/ASPS.2019.03.0301

Polycystic ovary syndrome (PCOS) is an endocrine disorder that influences women of reproductive age and may lead to serious complications. The symptoms of PCOS include irregular menstrual cycles, hirsutism, signs of hyperandrogenism and insulin resistance. The patients with PCOS are at increased risk for developing reproductive, metabolic and cardiovascular disorders [1]. Several contributing factors such as genetic, environmental factors, hormonal disturbances combine with other factors, including obesity, ovarian dysfunction and hypothalamic pituitary anomalies contribute to the etiology of PCOS. Although being one of the most common reproductive health problems of women, its effective treatment therapy remains a significant challenge to clinicians. Insulin resistance plays an important and prime role in the pathophysiology of this disorder, and for this the use of oral antidiabetics most compelling [2]. Hyperandrogenism and insulin resistance appear to be the main cause to the pathophysiology of this disoder. The glucose and insulin metabolism pathways have been elucidated and debated to understand whether Insulin Resistance is due to a defect in insulin action or a primary defect in $\beta$-cell function or decreased hepatic clearance of insulin, or a combination of all these factors. Various studies have reported that obese, normal weight and thin women with PCOS have a form of insulin resistance that is unique and intrinsic to the disorder. However obese women with PCOS possess an additional burden of insulin resistance resulting from their excess adiposity. Hyperinsulinemia causes increase in androgen production directly by acting as a co-gonadotropin, augmenting Luteinizing Hormone activity within the ovary, and indirectly by increasing serum LH pulse amplitude. Whereas Androgens may in turn contribute at least partially to the insulin resistance state linked with PCOS. PCOS is associated with significant reproductive morbidity including infertility, abnormal uterine bleeding, miscarriage and other complications of pregnancy during the reproductive years [3]. PCOS is not only an endocrine disease, but an amalgam of metabolic and psychosocial impairment. While further research needs to be done with bigger sample sizes, the current literature does suggest a decreased quality of life (QoL) in the population. Managing the symptoms of the patient seems like a very simple answer to this large problem; this will only occur if clinicians are committed to increase patient awareness, and in helping patients find the proper treatment for all aspects of this disorder. Early diagnosis and long term management can help control polycystic ovary syndrome so that women with polycystic ovary syndrome can still live a healthy active life and avoid longterm complications such as metabolic syndrome and cardiovascular diseases [4].

Therapeutic modalities of PCOS act to decrease hyperinsulinemia, restore fertility, treat hirsutism or acne, and correct menstrual irregularity. A lifestyle intervention that addresses healthy diet with caloric restriction, exercise to aid in weight loss and prevention of future weight gain and behavior change support is the best first-line treatment for PCOS. Future research has to aim on the missing chinks in our growing knowledge about this problem. Following those physicians will be able to provide the finest care for patients. PCOS has no up to the mark treatment till now and mostly patient gets only symptomatic treatment therapy with hormones and insulin sensitizers and patients becomes drug dependent in the long term. Unani physicians have recommended regular induction of menstruation, Correction in insulin levels etc. as treatment modalities [1].

\section{Bibliography}

1. Maqbool M., et al. "Polycystic ovarian syndrome- a multifaceted disease: a review”. International Journal of Pharmaceutical Sciences and Research 10.3 (2019): 1072-1079.

2. Mudasir Maqbool., et al. "Polycystic in Ovarian Syndrome and Its Various Treatment Strategies". Indo American Journal of Pharmaceutical Research 5 (2018): 8670-8678.

3. Maqbool M., et al. "Insulin Resistance and Polycystic ovary Syndrome: A Review". Journal of Drug Delivery and Therapeutics 9.1 (2019): 433-436

4. Brady C., et al. "Polycystic ovary syndrome and its impact on women's quality of life: More than just an endocrine disorder". Drug, Healthcare and Patient Safety 1 (2009): 9-15.

Volume 3 Issue 7 July 2019

(c) All rights are reserved by Mudasir Maqbool. 